\title{
Web Based Air Pollution Monitoring System (Air Pollution Monitoring Using Smart Phone)
}

\author{
Shilpa R. Khodve ${ }^{1}$, A. N. Kulkarni ${ }^{2}$ \\ ${ }^{1}$ Department of Electronics and Telecommunication, Zeal College of Engineering and Research, Pune, India \\ ${ }^{2}$ Professor, Department of Electronics and Telecommunication, Zeal College of Engineering and Research, Pune, India
}

\begin{abstract}
This system will have ARM7 LPC2138, which is heart of the system. Sensors like temperature, smoke, CO, NO are interfaced with microcontroller for the environmental monitoring air pollution. All the parameters of the sensors are displayed on the LCD. All the values are sent to the nearby mobile using Bluetooth. At the mobile android web page is designed to show the All parameters worldwide these values are display on the mobile window as well as worldwide mobile so that PC Data will be viewed by the any engineer from any end of the world by this system. this system will have one more facility as all the values are sent by microcontroller to the Mobile. Mobile application will note down the coordinates of the area with sensor values stored in the form of database and Person will be able to view the sensor vise air pollution area wise due to the GPS facility. One more system feature is that it will show the all the values of the sensor on the monitor window as well as these values are compared with threshold value of the air pollution If any of the value goes above the threshold then the system will send the message to the administrative part or the engineer.
\end{abstract}

Keywords: Web server, microcontroller,GPS,GSM,Android

\section{Introduction}

An air pollutant is a substance in the air that can have adverse effects on humans and the ecosystem. The substance can be solid particles, liquid droplets, or gases. A pollutant can be of natural origin or man-made. Pollutants are classified as primary or secondary. Primary pollutants are usually produced from a process, such as ash from a volcanic eruption. Other examples include carbon monoxide gas from motor vehicle exhaust, or the sulfur dioxide released from factories. Secondary pollutants are not emitted directly. Rather, they form in the air when primary pollutants react or interact. Ground level ozone is a prominent example of a secondary pollutant. Pollutants are either emitted directly or formed from other primary pollutants.

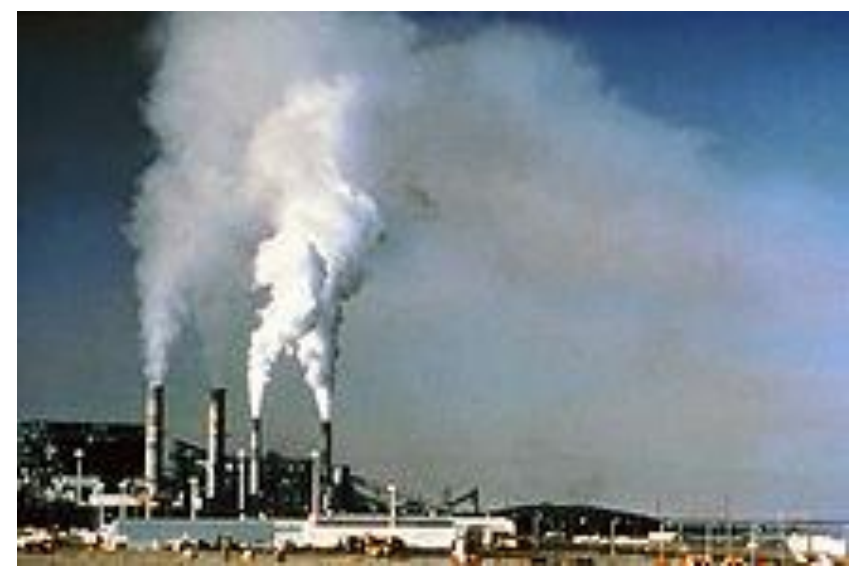

Figure 1

Before fuel gas desulfurization was installed, the emissions from this power plant in new-mexico contained excessive amounts of sulfur dioxide.

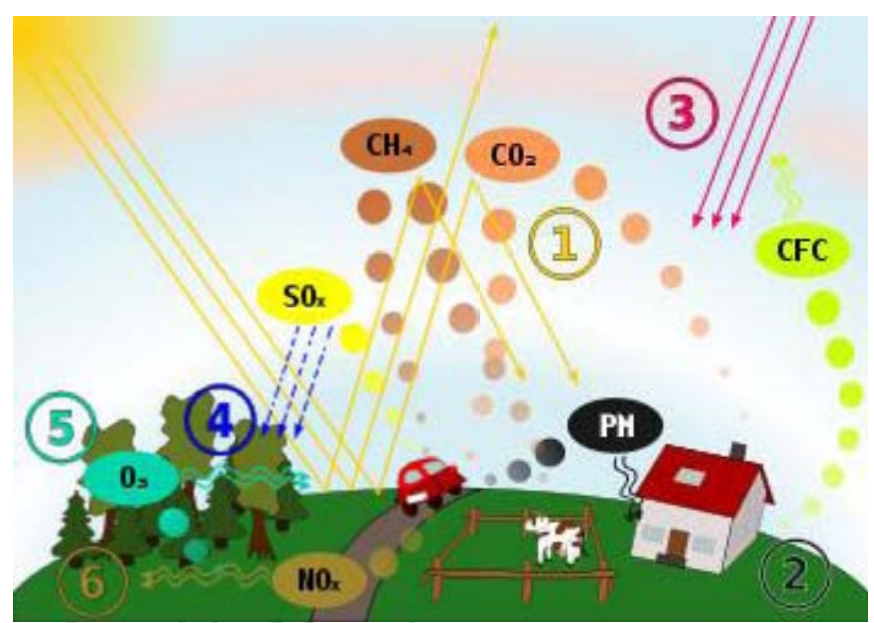

Figure 2

Schematic drawing, causes and effects of air pollution: (1) greenhouse effect, (2) particulate contamination, (3) increased UV radiation, (4) acid rain, (5) increased ground level ozone concentration, (6) increased levels of nitrogen oxides.

Major primary pollutants produced by human activity include:

- Sulfur oxides $\left(\mathrm{SO}_{\mathrm{x}}\right)$ - particularly sulfur dioxide, a chemical compound with the formula $\mathrm{SO}_{2}$. it is produced by volcanoes and in various industrial processes. Coal and petroleum often contain sulfur compounds, and their combustion generates sulfur dioxide. Further oxidation of $\mathrm{SO}_{2}$, usually in the presence of a catalyst such as $\mathrm{NO}_{2}$, forms $\mathrm{H}_{2} \mathrm{SO}_{4}$, and thus acid rain.[2] This is one of the causes for concern over the environmental impact of the use of these fuels as power sources.

- Nitrogen oxides $\left(\mathrm{NO}_{\mathrm{x}}\right)$ - Nitrogen oxides, particularly nitrogen dioxide, are expelled from high temperature combustion, and are also produced during thunderstorms by electric discharge. They can be seen as a brown haze dome above or a plume downwind of cities. Nitrogen dioxide is a chemical compound with the formula $\mathrm{NO}_{2}$. It 


\section{International Journal of Science and Research (IJSR) \\ ISSN (Online): 2319-7064 \\ Index Copernicus Value (2013): 6.14 | Impact Factor (2014): 5.611}

is one of several nitrogen oxides. One of the most prominent air pollutants, this reddish-brown toxic gas has a characteristic sharp, biting odor.

- Carbon monoxide (CO) - CO is a colorless, odorless, toxic yet non-irritating gas. It is a product by incomplete combustion of fuel such as natural gas, coal or wood. Vehicular exhaust is a major source of carbon monoxide.

- Volatile organic compounds (VOC) - VOCs are a wellknown outdoor air pollutant. They are categorized as either methane $\left(\mathrm{CH}_{4}\right)$ or non-methane (NMVOCs). Methane is an extremely efficient greenhouse gas which contributes to enhanced global warming. Other hydrocarbon VOCs are also significant greenhouse gases because of their role in creating ozone and prolonging the life of methane in the atmosphere. This effect varies depending on local air quality. The aromatic NMVOCs benzene, toluene and xylene are suspected carcinogens and may lead to leukemia with prolonged exposure. 1,3butadiene is another dangerous compound often associated with industrial use.

- Particulates, alternatively referred to as particulate matter (PM), atmospheric particulate matter, or fine particles, are tiny particles of solid or liquid suspended in a gas. In contrast, aerosol refers to combined particles and gas. Some particulates occur naturally, originating from volcanoes, dust storms, forest and grassland fires, living vegetation, and sea spray. Human activities, such as the burning of fossil fuels in vehicles, power plants and various industrial processes also generate significant amounts of aerosols. Averaged worldwide, anthropogenic aerosols - those made by human activities-currently account for approximately 10 percent of our atmosphere. Increased levels of fine particles in the air are linked to health hazards such as heart disease, ${ }^{[6]}$ altered lung function and lung cancer.

\section{Related Literature Survey}

An Environmental Air Pollution Monitoring System for monitoring the concentrations of major air pollutant gases has been designed, developed, and tested complying with the wireless standard. This system measures concentrations of gases such as $\mathrm{CO}, \mathrm{NO} 2$ and $\mathrm{SO} 2$, and using semiconductor sensors. The hardware unit integrates a single-chip microcontroller, air pollution sensors array, a GSM-Module and a GPS-Module. The Central-Server is a high-end personal computer application server with internet connectivity. The hardware unit gathers air pollutants levels (CO, NO2, and SO2), and packs them in a frame with the GPS physical location, time, and date. The frame is subsequently uploaded to the GSM-Modem and transmitted to the Central-Server via wireless network.[1] In this paper Environmental air pollution has significant influence on the concentration of constituents in the atmosphere leading to effects like global warming and acid rains. To avoid such adverse imbalances in the nature, an air pollution measuring system is utmost important. The traditional air quality monitoring system, controlled by the Pollution Control Department, is extremely expensive. Wireless Sensor Networks are a new and very challenging research field for embedded system design automation, as their design must enforce stringent constraints in terms of power and cost. This paper attempts to develop an effective solution for pollution measuring using wireless sensor networks (WSN).[2] In this paper it focuses on development of a prototype for a Wireless Sensor Network (WSN) that monitors various environmental parameters of interest in urban areas based on ZigBee protocol. This is performed through a small device that can be placed anywhere in a city. First, it is studied the operation of ZigBee protocol.Second, it was chosen and tested a ZigBee module and sensors from the market. Then, it was developed a module that monitors: humidity, temperature, light, carbon monoxide, carbon dioxide and oxygen. These data are measured and sent periodically to a base station connected to a computer. These data are stored and processed for presentation on the Internet [3] In this Environment Observation and Forecasting System (EOFS) is a application for monitoring and providing a forecasting about environmental phenomena. We design air pollution Monitoring system which involves a context model and a flexible data acquisition policy. The context model is used for understanding the status of air pollution on the remote Place. It can provide an alarm and safety guideline depending on the condition of the context model.It also supports the flexible sampling interval change for effective the tradeoff between sampling rates and battery lifetimes.[4] In this PolluMap is a new automated system that monitors the air quality of urban cities and displays the information using a web service. The system collects pollution data using mobile hardware modules, transmits the data regularly using GPRS to a back-end server, and integrates the data to generate a pollution map of the city using its geographical information system. The pollution map is available at anytime from an easy-to-view website.[5] The proposed system consists of a Mobile Data-Acquisition Unit(MobileDAQ) and a fixed Internet-Enabled Pollution Monitoring Server (Pollution-Server).The Mobile-DAQ unit integrates a single-chip microcontroller, air pollution sensors array, a General Packet Radio Service Modem (GPRS-Modem), and a Global Positioning System Module (GPS-Module). The Pollution-Server is a high-end personal computer application server with Internet connectivity.The Mobile-DAQ unit gathers air pollutants levels (CO,NO2, and SO2), and packs them in a frame with the GPS physical location, time, and date. The frame is subsequently uploaded to the GPRSModem and transmitted to the Pollution-Server via the public mobile network [6]

\section{Proposed System}

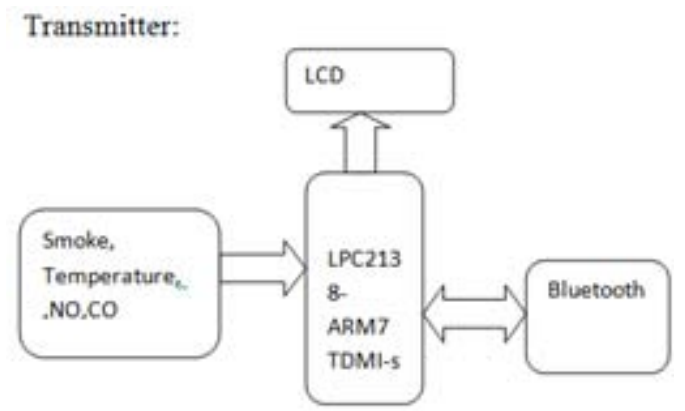




\section{International Journal of Science and Research (IJSR)

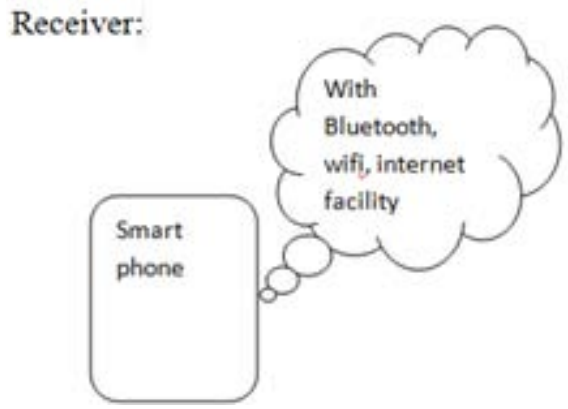

Figure 3: Block Diagram

A wireless sensor network (WSN) is an infrastructure comprised of sensing, computing and communication elements that allows the administrator to monitor \& control of the specified parameters in the network. Typical application of WSN includes data collection, monitoring, surveillance \& medical telemedicine. It is also used in irrigation system, Greenhouses for monitoring \& controlling parameters like water flow, temp, humidity, moisture, etc

\section{Developed System}

\section{Circuit Diagram Explanation}

- $\quad$ LPC 2138 is used as a processor in this system. Here we are connecting bletooth \& UART1 of LPC2138.

- MAX232 protocol is used for connecting Bluetooth modems to interface with LPC2138.

- In sensors CO sensor ,smoke sensor ,NH sensor, temperature sensor, humidity sensor are connected to the ADC pin of IC p0.28, p0.29, p0.30, p0.25, p0.21,

- 16x2 LCD display is used to display certain outputs such as temperature all the value of the Temp. sensor etc. LCD is interfaced with P0.16 to P0.19 and P0.20 $\mathrm{RS}$ and

- $\quad$ P0.22 -EN and r/w is GND.

- To provide power to the system 12 vis used as a power source, from which power output are $3.3 \mathrm{v} \& 5 \mathrm{v}$.

- $\quad$ LM1117 is used to deliver 3.3v to the LPC2138

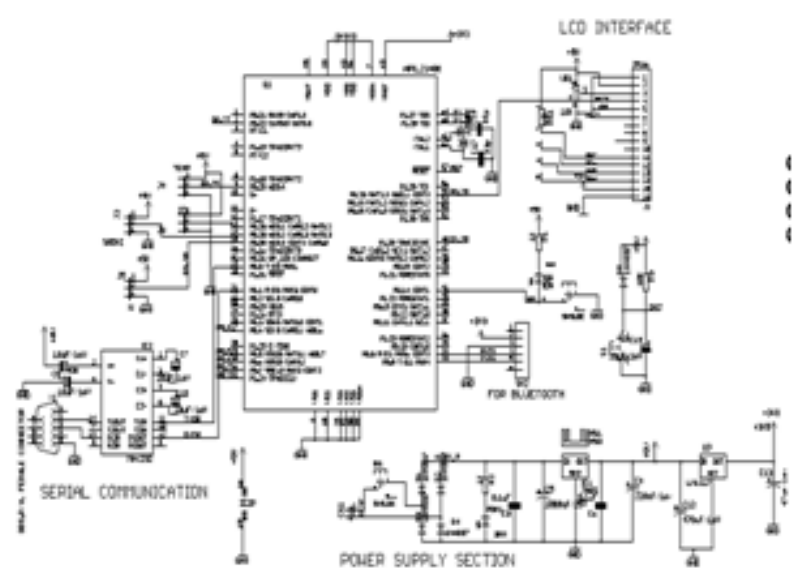

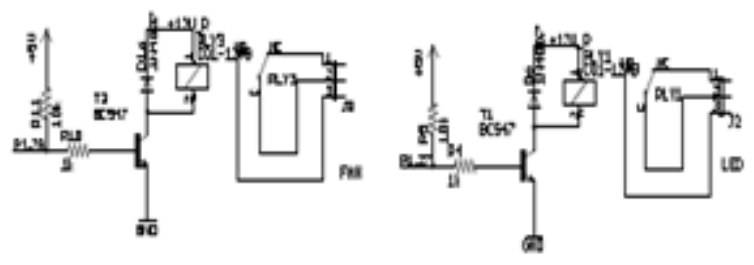

Figure 4 Circuit Diagram

\section{Conclusions}

The system utilizes city buses to collect pollutant gases such as $\mathrm{CO}, \mathrm{N}$, smoke and temperature. The data shows the pollutant levels and their to local air quality standards. Here successfully design the android web server which shows the real time air pollution data /air pollutants on web page which can be accessible from anywhere in the world so, here have designed circuit which takes corrective action on the increase of air pollution on the particular threshold value.

\section{References}

[1] A. R. Al-Ali, Imran Zualkernan and Fadi Aloul, -A Mobile GPRS-Sensors Array for Air Pollution Monitoring" IEEE SENSORS JOURNAL, VOL. 10, NO. 10, OCTOBER 2010

[2] Shashikant U.Suryawanshi, Deepganga Dhang ,Ashish A. Chougule Shailendra B. Mote, Implementation Of Embedded Wireless Air Pollution Monitoring System" IOSR Journal of Electronics and Communication Engineering (IOSR-JMCE) ISSN: 2278-2834-, ISBN: '2278-8735, PP: 27-30.

[3] Aruljothi.R , - AirPollution Measuring System with Mobile Sensor Arrays" International Journal of Scientific \& Engineering Research, Volume 4, Issue 5, May-2013 ISSN 2229-5518 .

[4] Rakesh Kumar Giri, -AnItinerant GPRS-GPS and Sensors Integration for Atmospheric Effluence Screening" International Journal of Technology And Engineering System(IJTES): Jan -March 2011Vol.2.No.2.

[5] N. Kularatna and B. H. Sudantha, -Anenvironmental air pollution monitoring system based on the IEEE 1451 standard for low cost requirements,"

[6] IEEE Sensors J., vol. 8, pp. 415-422, Apr. 2008.

[7] F. Tsow, E Forzani, A. Rai, R. Wang, R. Tsui, S. Mastroianni, C. Knobbe, A. J. Gandolfi, and N. J. Tao, - Awearable and wireless sensor system for real-time monitoring of toxic environmental volatile organic compounds," IEEE Sensors J., vol. 9, pp. 1734-1740, Dec. 2009.

[8] Y. J. Jung, Y. K. Lee, D. G. Lee, K. H. Ryu, and S. Nittel, - Airpollution monitoring system based on geosensor network," in Proc. IEEE Int. Geoscience Remote Sensing Symp., 2008, vol. 3, pp. 1370-1373.

[9] C. J. Wong, M. Z. MatJafri, K. Abdullah, H. S. Lim, and K. L. Low, Femporal air quality monitoring using surveillance camera," in Proc. IEEE Int. Geoscience and Remote Sensing Symp., 2007, pp. 2864-2868.

[10]M. Gao, F. Zhang, and J. Tian, Environmental monitoring system with wireless mesh network based on embedded system," in Proc. 5th IEEE Int. Symp. Embedded Computing, 2008, pp. 174-179. 


\section{International Journal of Science and Research (IJSR) \\ ISSN (Online): 2319-7064}

Index Copernicus Value (2013): 6.14 | Impact Factor (2014): 5.611

[11] W. Chung and C. H. Yang, - Rmote monitoring system with wireless sensors module for room environment," Sens. Actuators B, vol. 113, no. 1, pp. 35-42, 2009.

[12] J. W. Kwon, Y. M. Park, S. J. Koo, and H. Kim, Đesign of air pollution monitoring system using ZigBee networks for ubiquitous-city," in Proc. Int. Conf. Convergence Information Technology, 2007, pp.1024-1031.

[13] M. AbuJayyab, S. Al Ahdab, M. Taji, Z. Al Hamdani, and F. Aloul, Pollumap: A pollution mapper for cities," in Proc. IEEE Innovations in Information Technology Conf., Dubai, UAE, Nov. 2006, pp. 1-5.

[14] H. W. Huang, The HCS12/9S12: An Introduction to Hardware and Software Interfacing, 1st ed. Florence, KY: Thomson Delmar Learning, 2006.

[15] Alpha Sense Gas Sensor Datasheets and Speculations.[Online].Available:http://www.alphasense. com/alphasense_sensors/sulfur_dioxide_sensors.html

[16] National Marine Electronics Association Data. [Online]. Available:

http://www.gpsinformation.org/dale/nmea.htm

[17]GPRS-Modem Technology. [Online]. Available: http://www.comtechm2m.com/gprs-modem/gsm-gprsmodem.htm

[18] WampServer.[Online].Available: http://www.wampserver.com

[19] Air Quality Index, UAE, Dubai Municipality. [Online]. Available:https://portal.dm.gov.ae/AirQuality/Airqualit yindex.htm 